\title{
Calibração de câmara semiaberta estática para quantificação de amônia volatilizada do solo
}

\author{
Ednaldo da Silva Araújo(1), Tatiana Marsola(2), Mário Miyazawa( ${ }^{(3)}$, Luís Henrique de Barros Soares(1), \\ Segundo Urquiaga ${ }^{(1)}$, Robert Michael Boddey ${ }^{(1)}$ e Bruno José Rodrigues Alves ${ }^{(1)}$
}

\begin{abstract}
(1)Embrapa Agrobiologia, BR 465, Km 7, CEP 23890-000 Seropédica, RJ. E-mail: ednaldo@cnpab.embrapa.br, luis.henrique@cnpab.embrapa.br, urquiaga@cnpab.embrapa.br, bob@cnpab.embrapa.br, bruno@cnpab.embrapa.br (2)Universidade de São Paulo, Centro de Energia Nuclear na Agricultura, Avenida Centenário, no 303, Caixa Postal 96, CEP 13400-970 Piracicaba, SP. E-mail: tatianamarsola@gmail.com ${ }^{(3)}$ Instituto Agronômico do Paraná, Rodovia Celso Garcia Cid, Km 375, Caixa Postal 481, CEP 86047-902 Londrina, PR. E-mail: miyazawa@iapar.br
\end{abstract}

Resumo - O objetivo deste trabalho foi calibrar uma câmara prática e de baixo custo, para quantificar a amônia volatilizada do solo. A calibração da câmara foi realizada pela técnica do balanço do isótopo ${ }^{15} \mathrm{~N}$. A amônia volatilizada foi capturada por uma câmara semiaberta livre estática (SALE), confeccionada a partir de garrafa de plástico transparente de politereftalato de etileno (PET) de 2 L. O interior da câmara apresentava livre circulação de ar, e possuía uma lâmina de espuma de poliuretano, com $2,5 \mathrm{~cm}$ de largura e $25 \mathrm{~cm}$ de comprimento, pendurada verticalmente, com a extremidade inferior inserida em frasco com solução ácida, que mantinha a espuma saturada. A recuperação do ${ }^{15} \mathrm{~N}_{-} \mathrm{NH}_{3}$ no coletor aumentou de forma linear com o aumento da taxa de volatilização de $\mathrm{NH}_{3}$ do solo. Os testes de campo mostraram eficiência de $57 \%$ na recuperação do $\mathrm{N}-\mathrm{NH}_{3}$ volatilizado do solo. $\mathrm{O}$ intervalo de coletas das espumas pode variar de um a seis dias, sem comprometer a eficiência do sistema. Para estudos de campo, com uso do método proposto, o fator de correção de 1,74 deve ser usado para estimar a real taxa de volatilização de amônia do solo.

Termos para indexação: fator de emissão de amônia, isótopo ${ }^{15} \mathrm{~N}$, sistema coletor de $\mathrm{NH}_{3}$, ureia, volatilização de amônia.

\section{Calibration of a semi-opened static chamber for the quantification of volatilized ammonia from soil}

\begin{abstract}
The objective of this work was to calibrate a practical and low-cost chamber for the quantification of ammonia volatilization from soil. The chamber was calibrated using the ${ }^{15} \mathrm{~N}$ isotope balance technique. The volatilized ammonia was captured by a semi-opened free static chamber built with a $2 \mathrm{~L}$ ethylene polyterephthalate (PET) plastic bottle. The inner side of the chamber had free-air circulation and a stripe of polyurethane foam, with $2.5 \mathrm{~cm}$ wide and $25 \mathrm{~cm}$ long, hanged vertically with the lower end inserted in a bottle containing acid solution, which kept the foam moistened. The recovery of ${ }^{15} \mathrm{~N}$-ammonia by the chamber increased linearly with the increase in the rate of soil ammonia volatilization. Field tests showed an efficiency of $57 \%$ in the volatilized ammonia recuperation. The sampling interval of foam can vary from one to six days without impairing the system efficiency. For studies in the field using the proposed method, a correction factor of 1.74 should be used to estimate the real rate of ammonia volatilization from soil.
\end{abstract}

Indexed terms: Ammonia emission factor, ${ }^{15} \mathrm{~N}$ isotope, $\mathrm{NH}_{3}$ collecting system, urea, ammonia volatilization.

\section{Introdução}

A perda de nitrogênio dos fertilizantes tem consequências econômicas e ambientais indesejáveis (Bolan et al., 2004; Emmett, 2007). Há diferentes vias de perda de $\mathrm{N}$, das quais a volatilização da amônia $\left(\mathrm{NH}_{3}\right)$ pode ser a principal em solos agrícolas (Bouwmeester et al., 1985). A adequada quantificação do $\mathrm{N}$ perdido por volatilização de $\mathrm{NH}_{3}\left(\mathrm{~N}-\mathrm{NH}_{3}\right)$ pode contribuir para o desenvolvimento do fator de emissão de amônia do solo, tanto regional como nacionalmente, além de permitir identificar as melhores estratégias de manejo para reduzir as perdas de $\mathrm{N}$ por esse processo (Smith et al., 2007).

A quantificação do total de $\mathrm{NH}_{3}$ volatilizada do solo pode ser obtida por meio de métodos diretos e indiretos. A quantificação direta é feita com métodos micrometeorológicos e por sistemas de incubação classificados como: fechado estático (Volk, 1959; Sommer \& Olesen, 1991); fechado dinâmico 
(Kissel et al., 1977); e semiaberto estático (Nômmik, 1973), que são baseados na captura, em meio ácido, da amônia volatilizada do solo. A calibração desses métodos pode ser realizada pelo método indireto do balanço isotópico de ${ }^{15} \mathrm{~N}$ (Lara-Cabezas \& Trivelin, 1990).

Além dos métodos citados, vários outros são encontrados na literatura (McGinn \& Janzen, 1998; Loubet et al., 1999; Rochette et al., 2001). Entretanto, a maioria foi avaliada apenas em condições de laboratório (Smith et al., 2007). Além disso, esses métodos geralmente são sofisticados e apresentam elevado custo, o que dificulta sua utilização em condições de campo. Conforme relatado por Smith et al. (2007), o método para quantificar a volatilização de $\mathrm{N}-\mathrm{NH}_{3}$ deve ser de fácil reprodução. Esses autores compararam três técnicas nas seguintes câmeras: fechada estática, fechada dinâmica (com túnel de vento) e micrometeorológica, e observaram que a técnica da câmara fechada estática, a mais simples das três estudadas, subestimou a volatilização de $\mathrm{NH}_{3}$, quando comparada às demais. Quanto às técnicas de câmara fechada dinâmica e micrometeorológica, apesar dos bons resultados apresentados, são laboriosas e de custo elevado, o que dificulta sua utilização, principalmente em estudos que exigem grande número de repetições.

As câmaras estáticas, semiabertas e fechadas, são as mais simples e, portanto, muito utilizadas em condições de campo (Cantarella et al., 2003; Port et al., 2003; Van der Stelt et al., 2007; Mkhabela et al., 2008). Entretanto, essas câmaras subestimam a volatilização de $\mathrm{NH}_{3}$, e a taxa de recuperação pode ser variável.

De acordo com Lara-Cabezas et al. (1999), a câmara semiaberta estática proposta por Nômmik (1973) também apresenta limitações. Esses autores observaram que esse coletor, mesmo após modificações, apresentou taxa de recuperação que variou de 1 a $50 \%$ do total de $\mathrm{N}-\mathrm{NH}_{3}$ volatilizado. Assim, diante da importância da correta quantificação do $\mathrm{N}$ perdido por volatilização de $\mathrm{NH}_{3}$, alguns estudos vem sendo realizados no sentido de calibrar um sistema com alta eficiência, que seja prático e de baixo custo. O Instituto Agronômico do Paraná desenvolveu uma câmara coletora de $\mathrm{N}-\mathrm{NH}_{3}$ simples e de baixo custo, constituída de um corpo de garrafa de plástico tipo PET. Mesmo sem calibração feita em condições de campo, a câmara já vem sendo utilizada para quantificar a volatilização de amônia in situ (Guedes, 2005; Hungria et al., 2006).
A câmara influencia a ventilação, a temperatura e a umidade do solo e do ar no seu interior e, consequentemente, pode ter efeito sobre a volatilização de $\mathrm{N}^{-\mathrm{NH}_{3}}$, em comparação à que ocorre em condições naturais (Lara-Cabezas \& Trivelin, 1990). Portanto, para a calibração da câmara coletora de $\mathrm{NH}_{3}$ semiaberta, o uso do balanço do isótopo ${ }^{15} \mathrm{~N}$ é essencial (Lara-Cabezas \& Trivelin, 1990; Lara-Cabezas et al., 1999), já que a calibração com uso do ${ }^{15} \mathrm{~N}$ permite quantificar o total de $\mathrm{NH}_{3}$ volatilizado, sem a influência da câmara no processo de volatilização.

O objetivo deste trabalho foi calibrar uma câmara prática e de baixo custo, para quantificar a amônia volatilizada do solo.

\section{Material e Métodos}

Este trabalho foi realizado em parceria com a Embrapa Agrobiologia, Seropédica, RJ e o Iapar, Londrina, PR, onde uma primeira configuração de sistema semiaberto para a quantificação da volatilização de $\mathrm{N}_{-} \mathrm{NH}_{3}$ foi proposta. Os experimentos foram instalados na área experimental da Embrapa Agrobiologia. A câmara estudada, pela característica de livre fluxo de ar por seu interior, difere do sistema semiaberto proposto por Nômmik (1973), e foi denominada SALE (câmara semiaberta livre estática).

Acâmara coletora de amônia SALE foi confeccionada a partir de garrafa de plástico transparente de politereftalato de etileno (PET), com capacidade para $2 \mathrm{~L} \mathrm{e} \mathrm{com} \mathrm{área} \mathrm{de} 0,008 \mathrm{~m}^{2}$ (Figura 1). A base da garrafa PET foi removida e posicionada na parte superior, com auxílio de arame galvanizado e do anel da tampa da garrafa (tampa após a remoção da parte superior), que serviu como proteção contra a influência de chuvas. A abertura superior, para circulação de ar no interior da câmara, era de $2,1 \mathrm{~cm}$ de diâmetro. No interior da garrafa PET, foi feito o sistema absorvedor de amônia, constituído de uma lâmina de espuma de poliuretano $\left(0,017 \mathrm{~g} \mathrm{~cm}^{-3}\right)$ com $3 \mathrm{~mm}$ de espessura, $2,5 \mathrm{~cm}$ de largura e $25 \mathrm{~cm}$ de comprimento, suspensa verticalmente com o auxílio de um fio rígido de $1,5 \mathrm{~mm}$ de diâmetro. Em um frasco de plástico com capacidade para $50 \mathrm{~mL}$, suspenso pela extremidade inferior do fio rígido, foram adicionados $10 \mathrm{~mL}$ de solução de $\mathrm{H}_{2} \mathrm{SO}_{4}, 1 \mathrm{~mol} \mathrm{dm}^{-3}+$ glicerina $(2 \% \mathrm{v} / \mathrm{v})$. No momento do preparo do sistema absorvedor de $\mathrm{N}-\mathrm{NH}_{3}$, a lâmina 
de espuma foi acondicionada dentro do frasco com a solução ácida e, em seguida, comprimida de forma a absorver a maior parte dessa solução. A lâmina de espuma permaneceu no frasco fechado até o momento de seu posicionamento no interior do corpo da câmara (garrafa PET). Na instalação, a lâmina de espuma foi mantida com a extremidade inferior dentro do frasco de $50 \mathrm{~mL}$, de forma a evitar respingos da solução ácida no substrato, e a outra extremidade da espuma foi presa à parte superior do fio rígido, para mantê-la na posição vertical. Nessa ocasião, o fio rígido com o frasco de $50 \mathrm{~mL}$ e a espuma foram introduzidos no corpo do coletor pela base e pendurados na borda superior, suspensos a aproximadamente $1,5 \mathrm{~cm}$ da superfície do solo.

A calibração do coletor SALE foi realizada em duas condições: em casa de vegetação, em substrato de areia lavada e temperatura controlada por exaustores $\left(\sim 26^{\circ} \mathrm{C}\right)$; e em condições similares às de campo, em área coberta, com ventilação e temperatura ambiente $\left(25-32^{\circ} \mathrm{C}\right)$.

Em casa de vegetação, foram instalados dois experimentos, um para estudar a influência do intervalo de coleta das espumas com a amônia absorvida, e outro para estudar a eficiência do coletor SALE. Em ambos os experimentos, utilizaram-se bandejas de plástico com $52,5 \mathrm{~cm}$ de comprimento por $32,5 \mathrm{~cm}$ de largura, com $3 \mathrm{~kg}$ de substrato (areia lavada, livre de nitrogênio). Para favorecer a volatilização de amônia, o $\mathrm{pH}$ do substrato foi previamente aumentado, com a adição de $5 \mathrm{~g}$ de $\mathrm{CaCO}_{3}$ por bandeja, o que resultou em $\mathrm{pH}$ superior a 7,5, antes da adição do fertilizante nitrogenado. Nos dois experimentos em casa de vegetação, a fonte de $\mathrm{N}$ utilizada foi o sulfato de amônio, pois a areia lavada não possui urease, enzima responsável pela hidrólise da ureia.

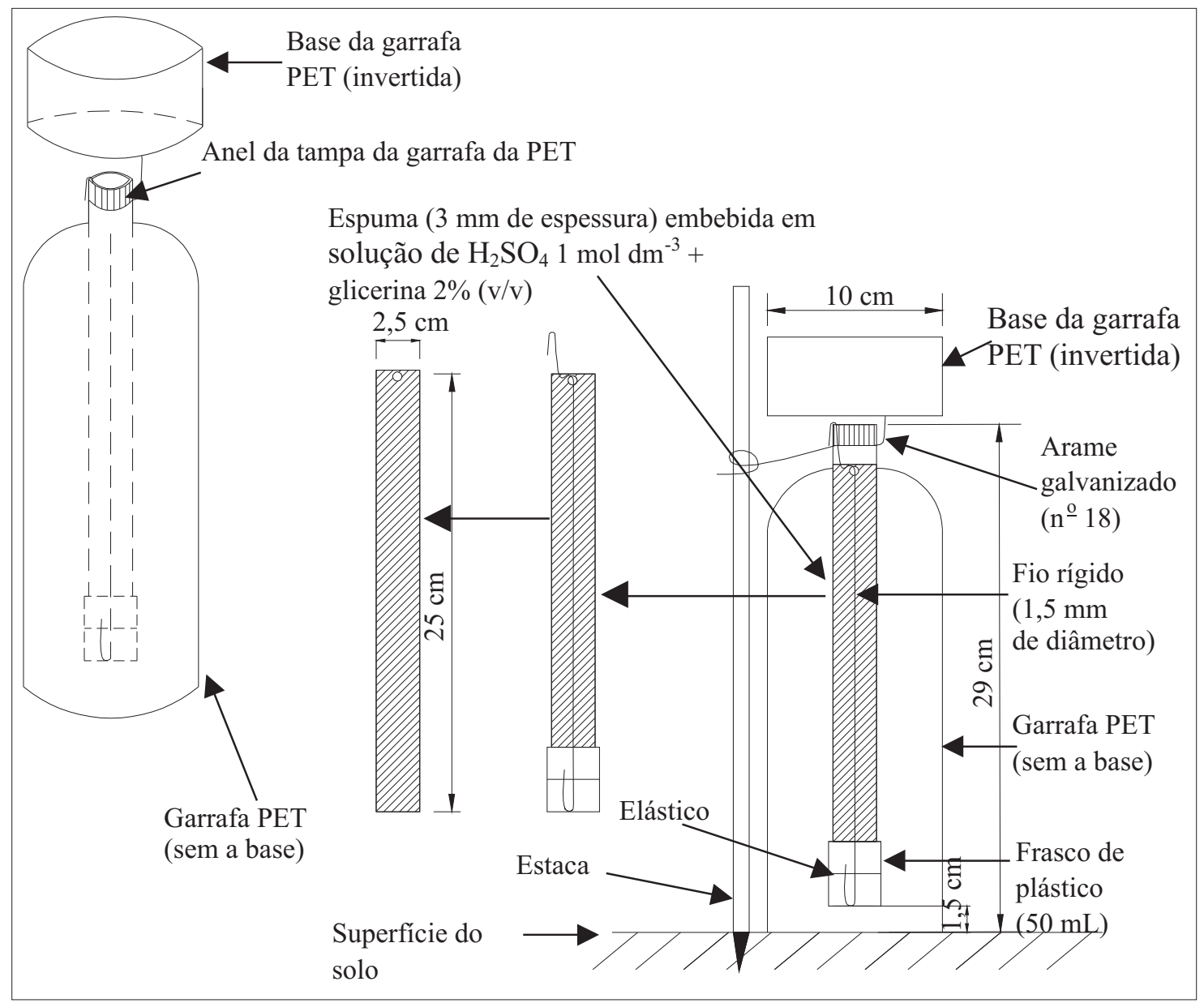

Figura 1. Câmara coletora de $\mathrm{NH}_{3}$ semiaberta livre estática (SALE). 
Para avaliar a influência dos períodos entre as coletas, os tratamentos consistiram de quatro intervalos diferentes, com 1, 2, 3 e 6 dias entre elas, e duas espessuras de espuma de poliuretano (3 e $7 \mathrm{~mm}$ ). Para cada tratamento, foram utilizadas três repetições distribuídas em blocos ao acaso. Na área total de cada bandeja, foi aplicada uma dose de $\mathrm{N}$ equivalente a $10 \mathrm{~g} \mathrm{~m}^{-2}\left(100 \mathrm{~kg} \mathrm{ha}^{-1}\right.$ de N). Após a última coleta, seis dias após a aplicação, coletaram-se amostras do substrato para determinação da umidade e do $\mathrm{N}$ remanescente. $\mathrm{O}$ nitrogênio volatilizado foi calculado pela diferença entre o $\mathrm{N}$ aplicado e o $\mathrm{N}$ remanescente em cada bandeja.

Para avaliar a eficiência do coletor SALE, em casa de vegetação, foram utilizadas seis doses de $\mathrm{N}$, aplicadas na forma de sulfato de amônio $(0,5,1$, 2, 4, 6 e $10 \mathrm{~g} \mathrm{~N} \mathrm{~m}^{-2}$ ), e um tratamento controle (vaso sem solo) para verificar possível contaminação entre as câmaras. $\mathrm{O}$ delineamento experimental utilizado foi o inteiramente casualizado, com três repetições. O sulfato de amônio foi diluído em $600 \mathrm{~mL}$ de água destilada e aplicado à superfície do substrato de forma homogênea, em toda a área da bandeja. Imediatamente após a adição do fertilizante, instalou-se um coletor SALE em cada bandeja. Efetuaram-se coletas das espumas com a amônia volatilizada do substrato, em intervalos de 24 horas, até completar 168 horas (7 dias). No penúltimo dia de coleta, após a troca das espuma, adicionaram-se $500 \mathrm{~mL}$ de $\mathrm{NaOH} 2 \mathrm{~mol} \mathrm{~L}^{-1}$ por bandeja, para favorecer a completa volatilização do $\mathrm{N}$ aplicado ao substrato.

No experimento em casa de vegetação, a eficiência do coletor SALE foi calculada de acordo com a equação Eal $(\%)=(\mathrm{Nva} / \mathrm{Na}) 100$, em que: Eal é a eficiência do sistema coletor SALE, em substrato de areia lavada; Nva é o $\mathrm{N}-\mathrm{NH}_{3}$ volatilizado e absorvido $\left(\mathrm{g} \mathrm{m}^{-2}\right)$; e $\mathrm{Na}$ é o N-sulfato de amônio total aplicado $\left(\mathrm{g} \mathrm{m}^{-2}\right)$, admitindose que todo o $\mathrm{N}$ aplicado foi volatilizado.

Para avaliar a eficiência do coletor SALE, em condições similares às de campo, foram utilizados vasos pequenos com $0,5 \mathrm{~kg}$ de terra, retirada da camada superficial $(0-20 \mathrm{~cm})$ de um Argissolo com textura média. $\mathrm{O}$ solo apresentou: $\mathrm{pH}\left(\mathrm{H}_{2} \mathrm{O}\right)$ 5,4; carbono orgânico de 1,25 $\mathrm{g} \mathrm{kg}^{-1}$; CTC de 11,61 $\mathrm{cmol}_{\mathrm{c}} \mathrm{dm}^{-3}$; saturação por bases de $41 \%$; e N total de $1,1 \mathrm{~g} \mathrm{~kg}^{-1}$. Para elevar o $\mathrm{pH}$, foi adicionado a cada vaso $1 \mathrm{~g}$ de carbonato de cálcio, o que resultou em $\mathrm{pH}$ em água de 7,5. O delineamento experimental adotado foi o inteiramente casualizado, com dois tratamentos: com e sem coletor SALE. Para evitar contaminação entre as câmaras, os vasos foram dispostos a cerca de $50 \mathrm{~cm}$ uns dos outros e também foram utilizadas câmaras sem aplicação de N, como controle. Para cada tratamento, foram usadas três repetições de seis doses de ureia, $(21,22 ; 42,45 ; 63,68 ; 84,90 ; 127,36$; e $169,81 \mathrm{mg}$ de $\mathrm{N}$ por vaso), com $1,3289 \%$ átomos de ${ }^{15} \mathrm{~N}$ em abundância, equivalente às adubações de 2,5, 5, 7,5, 10,15 , e $20 \mathrm{~g} \mathrm{~m}^{-2}$ considerando-se a superfície do vaso. O N-ureia foi aplicado em solução (20 mL por vaso). Para evitar perdas por desnitrificação, a umidade foi mantida a $50 \%$ da capacidade de campo e corrigida diariamente. Foi realizada a coleta das espumas com $\mathrm{NH}_{3}$ absorvido em três ocasiões, aos dois, cinco e nove dias da aplicação.

Cerca de $90 \%$ da volatilização de $\mathrm{N}-\mathrm{NH}_{3}$ ocorre nos primeiros seis dias da aplicação do N (Cantarella et al., 2003). Assim, ao final do experimento (nove dias após a aplicação do adubo), cada vaso recebeu $50 \mathrm{~mL}$ de $\mathrm{H}_{2} \mathrm{SO}_{4} 1 \mathrm{~mol} \mathrm{~L}^{-1}$, o que reduziu o $\mathrm{pH}$ do substrato para valores abaixo de 3,5 . Posteriormente, a terra de cada vaso foi seca à sombra e enviada ao laboratório para a determinação de ${ }^{15} \mathrm{~N}$ e $\mathrm{N}$ total. As análises de ${ }^{15} \mathrm{~N}$ foram feitas conforme Ramos et al. (2001).

Em todos os experimentos, a determinação do $\mathrm{N}-\mathrm{NH}_{3}$ retido na espuma foi realizada por destilação e titulação. A espuma e a solução, remanescentes em cada frasco de $50 \mathrm{~mL}$, foram transferidas para tubos de Erlenmeyer de $125 \mathrm{~mL}$, com massa previamente conhecida (P1). Posteriormente, adicionaram-se $40 \mathrm{~mL}$ de água destilada a cada tubo. Os tubos com a solução e a lâmina de espuma foram levados a um agitador horizontal a $220 \mathrm{rpm}$. Após $20 \mathrm{~min}$, os tubos foram removidos do agitador e pesados (P2). Uma alíquota de 5 a $10 \mathrm{~mL}$, conforme a concentração de $\mathrm{NH}_{4}{ }^{+}$esperada, foi analisada por destilação a vapor e submetida a titulação posterior, para a quantificação do $\mathrm{N}$ amoniacal conforme Alves et al. (1994). O volume total da solução foi determinado pela diferença entre $\mathrm{P} 2$ e P1 + massa da espuma. A densidade foi considerada igual a $1 \mathrm{~g} \mathrm{~cm}^{-3}$.

Em condições similares às de campo, a eficiência do coletor SALE foi obtida pela equação que relaciona a quantidade de $\mathrm{N}-\mathrm{NH}_{3}$ volatilizada do solo e proveniente do fertilizante, absorvida pelo coletor SALE (NVaPF), e a quantidade de $\mathrm{N}-\mathrm{NH}_{3}$ volatilizada proveniente do fertilizante, sem a influência do coletor (NVtPFsc), 
determinada pelo método do balanço de ${ }^{15} \mathrm{~N}$ no tratamento sem coletor.

Os dados experimentais foram submetidos à análise de variância e ao teste de Tukey, a 5\% de probabilidade. Nos estudos com aplicação de doses de $\mathrm{N}$ crescentes, foi realizada a análise de correlação entre as variáveis.

\section{Resultados e discussão}

A quantidade de $\mathrm{N}-\mathrm{NH}_{3}$ recuperada pelo coletor SALE não foi afetada pelos diferentes intervalos de coleta das espumas (Tabela 1). Da mesma forma, a espessura da espuma não afetou a recuperação do $\mathrm{N}-\mathrm{NH}_{3}$ volatilizado e, consequentemente, não afetou a eficiência do SALE em substrato de areia lavada (Eal). A espuma de $3 \mathrm{~mm}$ é a mais adequada, por facilitar o manuseio. De acordo com os resultados obtidos, a coleta da espuma pode ser realizada a intervalos que variam de um a seis dias. Para sistemas coletores baseados no de Nômmik (1973), as coletas podem ser realizadas a intervalos de três dias (Cantarella et al., 2003). Intervalos menores que 24 horas, provavelmente, não acarretariam em redução da eficiência, porém poderiam aumentar a mão de obra para a realização do estudo. Nos primeiros seis dias após a aplicação do fertilizante, recupera-se mais de $90 \%$ do $\mathrm{N}$ volatilizado proveniente do fertilizante aplicado (Cantarella et al., 2003). Assim, a partir da segunda semana da instalação do experimento, podem ser adotados intervalos maiores que os usados no início do trabalho, preferencialmente intervalos regulares.

Tabela 1. Eficiência da câmara coletora semiaberta livre estática (SALE), quanto à recuperação do nitrogênio volatilizado $\left(\mathrm{NH}_{3}\right)$ do substrato areia lavada, em condições de casa de vegetação, após aplicação de sulfato de amônio na dose $10 \mathrm{~g} \mathrm{~m}^{-2}$ de nitrogênio, em quatro intervalos de coleta e duas espessuras de espuma ${ }^{(1)}$.

\begin{tabular}{|c|c|c|c|c|c|c|}
\hline \multirow{2}{*}{$\begin{array}{l}\text { Intervalo de } \\
\text { coleta (dias) }\end{array}$} & \multicolumn{3}{|c|}{ Espuma de $3 \mathrm{~mm}$} & \multicolumn{3}{|c|}{ Espuma de $7 \mathrm{~mm}$} \\
\hline & $\begin{array}{c}\mathrm{NVt} \\
\left(\mathrm{g} \mathrm{m}^{-2}\right)\end{array}$ & $\begin{array}{l}\mathrm{NVa} \\
\left(\mathrm{g} \mathrm{m}^{-2}\right)\end{array}$ & $\begin{array}{l}\text { Eal } \\
(\%)\end{array}$ & $\begin{array}{c}\mathrm{NVt} \\
\left(\mathrm{g} \mathrm{m}^{-2}\right)\end{array}$ & $\begin{array}{c}\mathrm{NVa} \\
\left(\mathrm{g} \mathrm{m}^{-2}\right)\end{array}$ & $\begin{array}{l}\text { Eal } \\
(\%)\end{array}$ \\
\hline 1 & 6,96 & 5,19 & $74,8 \mathrm{a}$ & 6,66 & 4,97 & $73,67 a$ \\
\hline 2 & 6,75 & 5,55 & $81,4 a$ & 6,63 & 4,79 & $71,37 \mathrm{a}$ \\
\hline 3 & 6,81 & 5,07 & $76,0 \mathrm{a}$ & 6,49 & 4,98 & $75,03 \mathrm{a}$ \\
\hline 6 & 6,72 & 4,89 & $75,8 \mathrm{a}$ & 6,76 & 4,85 & $71,56 a$ \\
\hline Média geral & 6,81 & 5,17 & $77,0 \mathrm{~A}$ & 6,64 & 4,90 & $72,91 \mathrm{~A}$ \\
\hline
\end{tabular}

(1)Nvt, nitrogênio volatilizado do fertilizante, obtido pela diferença entre $\mathrm{N}$ aplicado e $\mathrm{N}$ remanescente em cada bandeja, após a última coleta; Nva, nitrogênio volatilizado do fertilizante absorvido no SALE; Eal (\%), eficiência do sistema coletor, dada por $100(\mathrm{NVa} / \mathrm{NVt})$. Médias seguidas de letras iguais, minúsculas nas colunas e maiúsculas nas linhas, não diferem significativamente pelo teste de Tukey, a 5\% de probabilidade.
Em casa de vegetação, a melhor correlação entre as

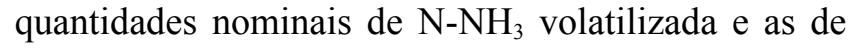
$\mathrm{N}-\mathrm{NH}_{3}$ absorvidas pelo sistema coletor foi obtida pelo modelo linear (Figura 2). De acordo com o modelo de ajuste, observou-se que a eficiência do coletor (inclinação da reta) não variou com o aumento da quantidade de $\mathrm{N}-\mathrm{NH}_{3}$ volatilizada. Deve-se destacar que a eficiência obtida não representa as interações que o $\mathrm{N}$-fertilizante pode sofrer, ao ser aplicado ao solo, uma vez que o substrato foi areia lavada e, assim, a única fonte de $\mathrm{N}$ foi o sulfato de amônio aplicado. Como os exaustores da casa de vegetação mantinham a circulação de ar interna, e o sistema coletor é semiaberto, era esperado que a eficiência ficasse abaixo de $100 \%$. Em condições sem ventilação, a eficiência pode ser maior do que a observada neste trabalho. Recomenda-se, portanto, para essas condições, realizar uma calibração para identificar a eficiência local.

Nômmik (1973) encontrou eficiência de 96\% para coletor semiaberto estático. Entretanto, Lara-Cabezas et al. (1999) alertam que coletores instalados sob a influência de uma fonte amoniacal isolada do meio, que force condições de perdas por elevação do $\mathrm{pH}$, podem não representar a volatilização de $\mathrm{NH}_{3}$ que ocorre em condições de campo, uma vez que o sistema coletor interfere no processo de volatilização. Assim, a Eal obtida no presente trabalho deve ser utilizada somente para estudos desenvolvidos em condições similares.

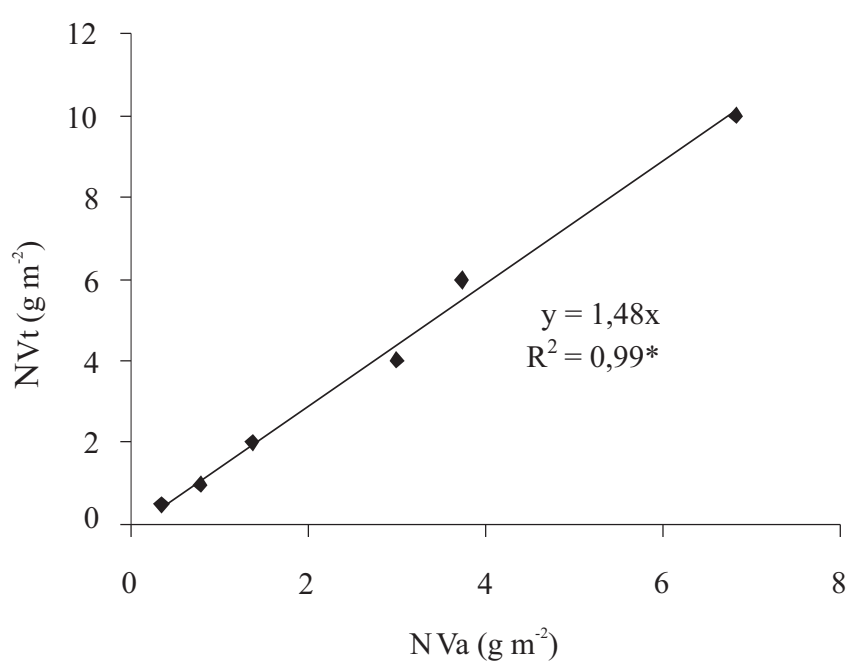

Figura 2. Regressão linear entre o nitrogênio volatilizado $\left(\mathrm{NH}_{3}\right)$ absorvido pelo coletor SALE (NVa) e o nitrogênio volatilizado total $(\mathrm{NVt})$, em condições de casa de vegetação. *Significativo a 5\% de probabilidade. 
A aplicação das diferentes doses de N-ureia não contribuiu para aumento significativo do teor de $\mathrm{N}$-total do solo (NtS) (Tabela 2), que tinha, originalmente, $569 \mathrm{mg}$ de N por $500 \mathrm{~g}$ de solo utilizados. Entretanto, a concentração de ${ }^{15} \mathrm{~N}$ no solo e a percentagem de $\mathrm{N}$ no solo proveniente do fertilizante (NSPF), como esperado, foram diferentes para cada dose aplicada. O coletor SALE não afetou a quantidade de $\mathrm{N}-\mathrm{NH}_{3}$ volatilizada proveniente do fertilizante (NVtPF). A volatilização do $\mathrm{N}$ aplicado foi de cerca de $46 \%$ nos dois tratamentos, com e sem coletor. No caso do coletor modificado por Lara-Cabezas \& Trivelin (1990), houve redução de $29 \%$ do N-ureia volatilizado, em relação ao tratamento sem coletor. Uma das diferenças entre os coletores usados naquele trabalho está na disposição da espuma que contém a solução absorvedora de $\mathrm{N}^{-\mathrm{NH}_{3}}$. Além disso, o sistema aqui apresentado era de livre circulação de ar, ao contrário dos sistemas baseados em Nômmik (1973). A disposição da espuma interfere na troca de ar do interior do coletor com a atmosfera. Assim, quanto maior for o impedimento para a troca de ar, maior será a redução da evaporação de água, o que favorecerá a reciclagem de água juntamente com o $\mathrm{NH}_{3}$ volatilizado do solo, pois $\mathrm{o} \mathrm{NH}_{3}$ é solúvel e possui alta afinidado pela água (McGinn \& Janzen, 1998).

$\mathrm{O}$ valor médio do $\mathrm{N}-\mathrm{NH}_{3}$ volatilizado absorvido proveniente do fertilizante (NVaPF) foi $89 \%$ do valor médio total do $\mathrm{N}_{-} \mathrm{NH}_{3}$ volatilizado absorvido no coletor SALE (NVa), ou seja, o $\mathrm{N}-\mathrm{NH}_{3}$ volatilizado absorvido proveniente do N-nativo do solo (NVaPS) correspondeu a $11 \%$ do NVa (Tabela 3). Entretanto, a percentagem de $\mathrm{N}$-nativo do solo no NVa variou com a dose de $\mathrm{N}$ aplicada. Na menor dose utilizada neste trabalho, $76 \%$ do NVa foi proveniente do fertilizante, e $24 \%$ foi proveniente do N-nativo do solo. Entretanto, na maior dose, a participação do N-nativo no NVa foi de apenas $5 \%$. Esses resultados mostram que, em estudos desta natureza, a utilização de tratamento controle, sem adubação, induz a resultados superestimados do $\mathrm{N}$ proveniente do fertilizante, por não estimar corretamente a volatilização do N-nativo do solo. Esses resultados estão de acordo com os obtidos por Lara-Cabezas \& Trivelin (1990) e Lara-Cabezas et al. (1999). De acordo com esses autores, a elevação do pH, pela hidrólise da ureia, afeta a atividade microbiana e, consequentemente, o processo de mineralização do N-nativo do solo.

Entre os resultados de $\mathrm{N}-\mathrm{NH}_{3}$ volatilizado total proveniente do fertilizante (NVtPF), obtidos do balanço de ${ }^{15} \mathrm{~N}$ no tratamento sem o coletor, e os de $\mathrm{N}-\mathrm{NH}_{3}$ proveniente do fertilizante absorvido pelo coletor SALE (NVaPF), a melhor correlação foi obtida por um modelo linear (Figura 3). Esses dados mostram que, assim como observado em casa de vegetação, a eficiência da câmara coletora de $\mathrm{NH}_{3}$ SALE não varia significativamente com as doses de $\mathrm{N}$ aplicadas.

Tabela 2. Nitrogênio total em $500 \mathrm{~g}$ de solo (NtS), concentração de ${ }^{15} \mathrm{~N}$ em excesso no solo, nitrogênio no solo proveniente do fertilizante (NSPF) e nitrogênio volatilizado $\left(\mathrm{NH}_{3}\right)$ total proveniente do fertilizante (NVtPF), após nove dias da aplicação de doses de $\mathrm{N}$ (mg por vaso) na forma de ureia ${ }^{(1)}$, com e sem o uso do coletor SALE ${ }^{(2)}$.

\begin{tabular}{|c|c|c|c|c|c|c|}
\hline Doses de $\mathrm{N}$ & $\begin{array}{c}\text { NtS } \\
\text { (mg por vaso) }\end{array}$ & $\begin{array}{c}\text { Excesso }{ }^{15} \mathrm{~N} \\
(\%)\end{array}$ & $\begin{array}{c}\text { NSPF } \\
\text { (mg por vaso) }\end{array}$ & $\begin{array}{c}\text { NSPF } \\
(\%) \\
\end{array}$ & $\begin{array}{c}\text { NVtPF } \\
\text { (mg por vaso) }\end{array}$ & $\begin{array}{c}\text { NVtPF } \\
(\%)\end{array}$ \\
\hline \multicolumn{7}{|c|}{ Com coletor SALE } \\
\hline 21,23 & $577,5 \mathrm{a}$ & $0,02148 f$ & $12,93 f$ & $2,24 \mathrm{f}$ & $8,30 \mathrm{f}$ & $39,1 b$ \\
\hline 42,45 & $568,3 \mathrm{a}$ & $0,03901 \mathrm{e}$ & $23,09 \mathrm{e}$ & $4,07 \mathrm{e}$ & $19,36 \mathrm{e}$ & $45,6 a b$ \\
\hline 63,68 & $607,1 \mathrm{a}$ & $0,05577 \mathrm{~d}$ & $35,30 \mathrm{~d}$ & $5,81 \mathrm{~d}$ & $28,38 d$ & $44,6 a b$ \\
\hline 84,91 & $591,3 a$ & $0,07018 \mathrm{c}$ & $41,96 \mathrm{c}$ & $7,31 \mathrm{c}$ & $42,94 \mathrm{c}$ & $50,6 \mathrm{a}$ \\
\hline 127,36 & $604,2 \mathrm{a}$ & $0,10356 b$ & $65,14 b$ & $10,79 b$ & $62,22 b$ & $48,9 \mathrm{a}$ \\
\hline 169,81 & $633,4 a$ & $0,12421 \mathrm{a}$ & $81,98 \mathrm{a}$ & $12,94 \mathrm{a}$ & $87,83 \mathrm{a}$ & $51,7 \mathrm{a}$ \\
\hline Média & - & - & $43,3 \mathrm{~A}$ & - & $41,5 \mathrm{~A}$ & $46,7 \mathrm{~A}$ \\
\hline $\mathrm{CV}(\%)$ & 4,57 & 2,99 & 5,08 & 3,37 & 5,32 & 5,50 \\
\hline \multicolumn{7}{|c|}{ Sem coletor SALE } \\
\hline 21,23 & $602,8 \mathrm{a}$ & $0,02139 f$ & $12,74 f$ & $2,24 \mathrm{f}$ & $8,49 f$ & $40,0 \mathrm{~b}$ \\
\hline 42,45 & $587,6 \mathrm{a}$ & $0,03901 \mathrm{e}$ & $24,51 \mathrm{e}$ & $4,07 \mathrm{e}$ & $17,94 \mathrm{e}$ & $42,3 \mathrm{ab}$ \\
\hline 63,68 & $610,4 a$ & $0,05585 \mathrm{~d}$ & $34,17 d$ & $5,81 \mathrm{~d}$ & $29,51 d$ & $46,3 \mathrm{ab}$ \\
\hline 84,91 & $625,6 a$ & $0,07040 \mathrm{c}$ & $44,71 \mathrm{c}$ & $7,32 \mathrm{c}$ & $40,19 c$ & $47,3 \mathrm{ab}$ \\
\hline 127,36 & $654,5 \mathrm{a}$ & $0,10440 b$ & $67,92 \mathrm{~b}$ & $10,85 b$ & $59,44 b$ & $46,7 \mathrm{ab}$ \\
\hline 169,81 & $639,6 a$ & $0,12502 \mathrm{a}$ & $85,03 \mathrm{a}$ & $12,99 \mathrm{a}$ & $84,78 \mathrm{a}$ & $49,9 a$ \\
\hline Média & - & - & $44,8 \mathrm{~A}$ & - & $40,1 \mathrm{~A}$ & $45,4 \mathrm{~A}$ \\
\hline CV $(\%)$ & 4,38 & 3,06 & 7,21 & 3,18 & 8,07 & 6,49 \\
\hline
\end{tabular}

${ }^{(1)}$ Enriquecida com $1,3289 \%$ de átomos de $15 \mathrm{~N}$ em abundância. ${ }^{(2)}$ Médias seguidas de letras iguais, minúsculas nas colunas e maiúsculas nas linhas, não diferem entre si pelo teste de Tukey, a 5\% de probabilidade. 
Tabela 3. Nitrogênio volatilizado $\left(\mathrm{NH}_{3}\right)$ total absorvido pelo coletor SALE (NVa), após dois, cinco e nove dias da aplicação de doses de $\mathrm{N}$ (mg por vaso) na forma de ureia ${ }^{(1)}$, concentração de ${ }^{15} \mathrm{~N}$ em excesso no $\mathrm{N}$ volatilizado, e nitrogênio volatilizado absorvido proveniente do fertilizante (NVaPF) e do solo (NVaPS), após nove dias da aplicação das doses de N.

\begin{tabular}{|c|c|c|c|c|c|c|c|c|}
\hline \multirow[t]{2}{*}{ Doses de $\mathrm{N}$} & \multicolumn{3}{|c|}{ NVa acumulado (mg por vaso) } & \multirow{2}{*}{$\begin{array}{c}\text { Excesso }{ }^{15} \mathrm{~N} \\
(\%)\end{array}$} & \multirow{2}{*}{$\begin{array}{c}\text { NVaPF } \\
\text { (mg por vaso) }\end{array}$} & \multirow{2}{*}{$\begin{array}{c}\text { NVaPF } \\
(\%)\end{array}$} & \multirow{2}{*}{$\begin{array}{c}\text { NVaPS } \\
\text { (mg por vaso) }\end{array}$} & \multirow{2}{*}{$\begin{array}{c}\text { NVaPS } \\
(\%)\end{array}$} \\
\hline & 2 dias & 5 dias & 9 dias & & & & & \\
\hline 21,23 & 2,84 & 3,81 & 4,29 & 0,7363 & 3,28 & 76,41 & 1,01 & 23,59 \\
\hline 42,45 & 7,67 & 9,97 & 11,10 & 0,8581 & 9,89 & 89,10 & 1,21 & 10,90 \\
\hline 63,68 & 9,52 & 13,36 & 15,00 & 0,8789 & 13,69 & 91,27 & 1,31 & 8,73 \\
\hline 84,91 & 14,25 & 19,86 & 21,97 & 0,8885 & 20,27 & 92,28 & 1,70 & 7,72 \\
\hline 127,36 & 18,57 & 28,68 & 32,29 & 0,9017 & 30,24 & 93,65 & 2,05 & 6,35 \\
\hline 169,81 & 28,41 & 44,79 & 50,71 & 0,9114 & 48,01 & 94,67 & 2,70 & 5,33 \\
\hline $\mathrm{CV}(\%)$ & 18,42 & 11,53 & 12,19 & 3,29 & 12,22 & 5,92 & 11,96 & 9,72 \\
\hline
\end{tabular}

${ }^{(1)}$ Enriquecida com $1,3289 \%$ de átomos $15 \mathrm{~N}$ em abundância.

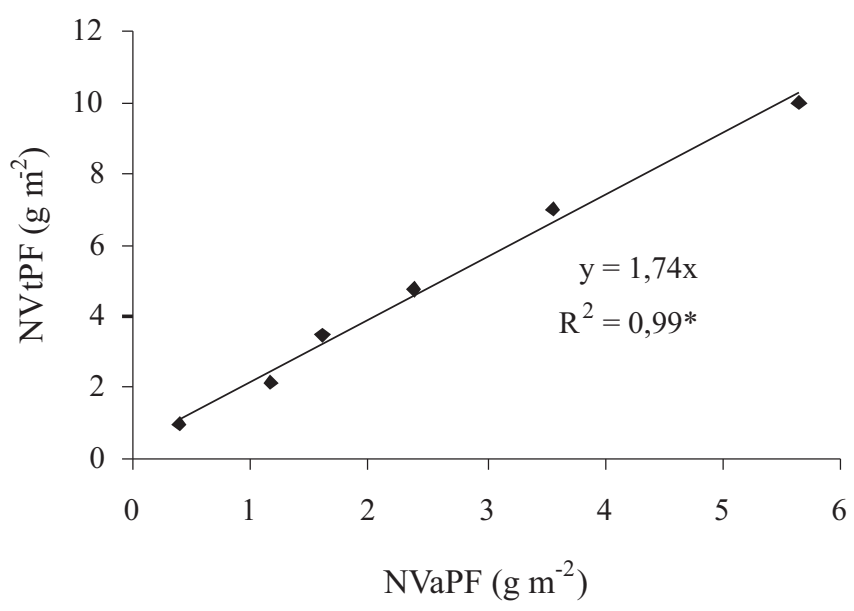

Figura 3. Regressão linear entre valores médios do nitrogênio volatilizado $\left(\mathrm{NH}_{3}\right)$ proveniente do fertilizante (NVaPF), absorvido pela câmara SALE, e do $\mathrm{NH}_{3}$ total proveniente do fertilizante (NVtPF), obtido pelo balanço de ${ }^{15} \mathrm{~N}$ no tratamento sem coletor. ${ }^{*}$ Significativo a $5 \%$ de probabilidade.

Lara-Cabezas \& Trivelin (1990) utilizaram doses de $\mathrm{N}$ equivalentes às taxas de adubação de 2, 4, 6 e $10 \mathrm{~g} \mathrm{~m}^{-2}$ e obtiveram quantidades de $\mathrm{N}$ volatilizado que se ajustaram a um modelo exponencial, tendo mostrado que, para o coletor semiaberto estático que utilizaram, a eficiência varia com a quantidade de $\mathrm{N}-\mathrm{NH}_{3}$ volatilizada.

Em razão do comportamento linear encontrado (Figura 3), a relação entre NVaPF e NVtPFsc foi representada pela equação $y=1,74 x+0,44$, em que o intercepto não difere de zero. Assim, o inverso do coeficiente angular é igual à eficiência do coletor SALE que, em condições similares às de campo, foi de $57 \%$ do total de $\mathrm{N}-\mathrm{NH}_{3}$ volatilizado do solo. Essa recuperação foi $37 \%$ superior à observada por
Lara-Cabezas et al. (1999). Além disso, como a eficiência do sistema não variou com a quantidade de $\mathrm{N}$ volatilizada, a quantidade real de $\mathrm{N}$ perdido por volatilização de $\mathrm{NH}_{3}$ foi obtida com o uso do fator 1,74. Para estudos em condições de campo, onde nem sempre a fonte de $\mathrm{N}$ tem distribuição uniforme, e considerando-se que o coletor SALE possui diâmetro de apenas $10 \mathrm{~cm}$, a instalação do coletor poderá ocorrer em microparcelas, nas quais a fonte de $\mathrm{N}$ tenha distribuição uniformizada.

\section{Conclusões}

1. A eficiência da câmara coletora semiaberta livre estática é mantida com o aumento da taxa de volatilização de $\mathrm{NH}_{3}$, tanto em condições de casa de vegetação como em condições similares às de campo.

2. A coleta das espumas pode ser realizada a intervalos entre 1 e 6 dias, sem comprometer a eficiência do sistema.

3. Para estudos de campo com uso do método proposto, o fator de correção 1,74 deve ser usado para estimar a real taxa de volatilização de amônia do solo.

\section{Agradecimentos}

À Fundação Carlos Chagas Filho de Amparo à Pesquisa do Estado do Rio de Janeiro, à Coordenação de Aperfeiçoamento de Pessoal de Nível Superior e ao Conselho Nacional de Desenvolvimento Científico e Tecnológico, por concessão de bolsas; a Agnê Morais Duarte, pela contribuição no desenho do coletor SALE; à Embrapa Agrobiologia e à Universidade Federal Rural do Rio de Janeiro, pelo apoio com infraestrutura necessária ao trabalho. 


\section{Referências}

ALVES, B.J.R.; SANTOS, J.C.F. dos; URQUIAGA, S.; BODDEY, R.M. Métodos de determinação do nitrogênio em solo e planta. In: HUNGRIA, M.; ARAÚJO, R.S. (Ed.). Manual de métodos empregados em estudos de microbiologia agrícola. Brasília: Embrapa-SPI, 1994. p.449-469.

BOLAN, N.S.; SAGGAR, S.; LUO, J.F.; BHANDRAL, R.; SINGH, J. Gaseous emission of nitrogen from grazed pastures: processes, measurements and modelling, environmental implications, and mitigation. Advances in Agronomy, v.84, p.37-120, 2004.

BOUWMEESTER, R.J.B.; VLEK, P.L.G.; STUMPE, J.M. Effect of environmental factors on ammonia volatilization from urea-fertilized soils. Soil Science Society of America Journal, v.49, p.376-381, 1985.

CANTARELLA, H.; MATOS, D.; QUAGGIO, J.A.; RIGOLIN, A.T. Fruit yield of 'Valencia' sweet orange fertilized with different $\mathrm{N}$ sources and the loss of applied N. Nutrient Cycling in Agroecosystems, v.67, p.215-223, 2003.

EMMETT, B.A. Nitrogen saturation of terrestrial ecosystems: some recent findings and their implications for our conceptual framework. Water, Air and Soil Pollution: Focus, v.7, p.99-109, 2007.

GUEDES, C.A.B. Volatilização de $\mathbf{N}$ e alterações químicas do solo sob cultivo de cana-de-açúcar com aplicação de vinhaça e diferentes formas de colheita. 2005. 78p. Dissertação (Mestrado) - Universidade Federal Rural do Rio de Janeiro, Seropédica.

HUNGRIA, M.; FRANCHINI, J.C.; CAMPO, R.J.; CRISPINO, C.C.; MORAES, J.Z.; SIBALDELLI, R.N.R.; MENDES, I.C.; ARIHARA, J. Nitrogen nutrition of soybean in Brazil: contributions of biological $\mathrm{N}_{2}$ fixation and $\mathrm{N}$ fertilizer to grain yield. Canadian Journal of Plant Science, v.86, p.927-939, 2006.

KISSEL, D.E.; BREWER, H.L.; ARKIN, G.F. Design and test of a field sampler for ammonia volatilization. Soil Science Society of America Journal, v.41, p.1133-1138, 1977.

LARA-CABEZAS, W.A.R.; TRIVELIN, P.C.O. Eficiência de um coletor semi-aberto estático na quantificação de $\mathrm{N}-\mathrm{NH}_{3}$ volatilizado da uréia aplicada ao solo. Revista Brasileira de Ciência do Solo, v.14, p.345-352, 1990.

LARA-CABEZAS, W.A.R.; TRIVELIN, P.C.O.; BENDASSOLLI, J.A.; SANTANA, D.G. de; GASCHO, G.J. Calibration of a semi-open static collector for determination of ammonia volatilization from nitrogen fertilizers. Communication in Soil Science and Plant Analysis, v.30, p.389-406, 1999.
LOUBET, B.; CELLIER, P.; FLURA, D.; GÉNERMONT, S. An evaluation of the wind tunnel technique for estimating ammonia volatilization from land. Part I. Analysis and improvement accuracy. Journal of Agricultural Engineering Research, v.72, p.71-81, 1999.

MCGINN, S.M.; JANZEN, H.H. Ammonia sources in agriculture and their measurement. Canadian Journal of Soil Science, v.78, p.139-148, 1998.

MKHABELA, M.S.; MADANI, A.; GORDON, R.; BURTON, D.; CUDMORE, D.; ELMI, A.; HART, W. Gaseous and leaching nitrogen losses from no-tillage and conventional tillage systems following surface application of cattle manure. Soil \& Tillage Research, v.98, p.187-199, 2008.

NÔMMIK, H. The effect of pellet size on the ammonia loss from urea applied to forest soils. Plant and Soil, v.39, p.309-318, 1973.

PORT, O.; AITA, C.; GIACOMINI, S.J. Perda de nitrogênio por volatilização de amônia com o uso de dejetos de suínos em plantio direto. Pesquisa Agropecuária Brasileira, v.38, p.857-865, 2003.

RAMOS, M.G.; VILLATORO, M.A.A.; URQUIAGA, S.;ALVES, B.J.R.; BODDEY, R.M. Quantification of the contribution of biological nitrogen fixation to tropical green manure crops and the residual benefit to a subsequent maize crop using ${ }^{15} \mathrm{~N}$-isotope techniques. Journal of Biotechnology, v.91, p.105-115, 2001.

ROCHETTE, P.; CHANTIGNY, M.H.; ANGERS, D.A.; BERTRAND, N.; COTE, D. Ammonia volatilization and soil nitrogen dynamics following fall application of pig slurry on canola crop residues. Canadian Journal of Soil Science, v.81, p.515-523, 2001.

SMITH, E.; GORDON, R.; BOURQUE, C.; CAMPBELL, A. Comparison of three simple field methods for ammonia volatilization from manure. Canadian Journal of Soil Science, v.87, p.469-477, 2007.

SOMMER, S.G.; OLESEN, J.E. Effects of dry matter content and temperature on ammonia loss from surface-applied cattle slurry. Journal of Environmental Quality, v.20, p.679- 683, 1991.

VAN DER STELT, B.; TEMMINGHOFF, E.J.M.; VAN VLIET, P.C.J.; VAN RIEMSDIJK, W.H. Volatilization of ammonia from manure as affected by manure additives, temperature and mixing. Bioresource Technology, v.98, p.3449-3455, 2007.

VOLK, M.G. Volatile loss of ammonia following surface application of urea to turf of bare soils. Agronomy Journal, v.51, p.746-749, 1959.

$\overline{\text { Recebido em } 30 \text { de janeiro de } 2009 \text { e aprovado em } 1 \text { de julho de } 2009}$ 